\title{
A summary about relaxin and its reproductive function
}

\begin{abstract}
Relaxin is a pleiotropic hormone included in the insulin-like-growth factor family (IGF) that is produced in a variety of reproductive tissues including corpus luteum, uterus, testis, prostate and others. Relaxin facilitates parturition, participates in uterine contractility, promotes angiogenesis, plays a role in spermatogenesis or promotes spermatozoa motility and acrosome reaction, among others physiological functions. In this review, we will focus on the principal roles of relaxin in female and male reproduction.
\end{abstract}

Keywords: relaxin, reproduction, parturition, pregnancy, spermatogenesis, male reproduction, angiogenesis
Volume 4 Issue 5 - 2017

\author{
Alana Aragon-Herrera,' Sandra Feijoo- \\ Bandin, 1,2 Diego Rodriguez-Penas,' 'Manuel \\ Portoles, ${ }^{2,3}$ Esther Rosello-Lleti, ${ }^{2,3}$ Miguel \\ Rivera, ${ }^{2,3}$ Jose Ramon Gonzalez-Juanatey, ${ }^{1,2}$ \\ Francisca Lago ${ }^{1,2}$ \\ 'Cellular and Molecular Cardiology Research Unit and \\ Department of Cardiology of Institute of Biomedical Research \\ and University Clinical Hospital, Spain \\ ${ }^{2}$ Centro de Investigacion Biomedica en Red de Enfermedades \\ Cardiovasculares, Spain \\ ${ }^{3}$ La Fe University Hospital, Spain
}

\begin{abstract}
Correspondence: Alana Aragón-Herrera, Unidad de Investigación en Cardiología Celular y Molecular (7), Instituto de Investigaciones Sanitarias de Santiago de Compostela (IDIS), Planta -2, Edificio de Consultas Externas, Hospital Clínico Universitario, Travesía Choupana s/n, 15706 Santiago de Compostela, Spain, Tel + 34981955089 ,

Email alannah.aragon@gmail.com
\end{abstract}

Received: April 26, 2017 | Published: May 10, 2017
Abbreviations: IGF, insulin-like-growth factor family; VEGF, vascular endothelial growth factor; RXFP1, relaxin/insulin-like family peptide receptor 1; INSL3, insulin-like peptide 3; cAMP, cyclic adenosine monophosphate INSL, insulin-like peptide

\section{Introduction}

Relaxin is a peptide hormone that is present in mammals and is secreted mainly by the corpus luteum during gestation. Relaxin belongs to the insulin-like-growth factor family (IGF) because of its similarity to the insulin structure. There are seven peptides in the relaxin family (relaxin 1, 2, 3 and INSL (insulin-like peptide) 3, 4, 5 and 6) $\cdot{ }^{1-3}$ Relaxin is a pleiotropic hormone with effects at different physiological levels, especially in reproduction. In 1926, Frederick Hisaw discovered its role in the expansion of the pubic ligament to adapt birth canal and facilitate parturition in female. ${ }^{2,4}$ In terms of reproduction, relaxin-1 and relaxin-2 expression is detected in different reproductive tissues: in females in uterus, corpus luteum, secretory endometrium, decidualized stromal cells of the endometrium, mammary gland, and in males in testis and prostate..$^{2,4}$ The principal physiological functions of relaxin in reproduction include the softening of the pubic ligament, the promotion of endometrial vascularization and the readjustment of connective tissue. ${ }^{3}$ The hormone relaxin is also produced in many tissues like the heart, peripheral blood, brain, lung, kidney, liver, adipose tissue and pancreas. ${ }^{2}$

\section{Relaxin and reproduction in females}

During pregnancy, relaxin has endocrine roles and is highly secreted from different sources depending of the species. The principal role of relaxin is to increase the elasticity and flexibility of pubic ligament in order to facilitate the birth of the fetus. ${ }^{2}$ In humans, circulating relaxin is mainly released by the corpus luteum, but Northern blotting assays have shown relaxin expression in placenta, placental trophoblast, amnion, chorion, basal plate and decidua parietalis. In the initial stages of pregnancy in humans, relaxin reaches high levels in the first trimester but then decline, while in mice, rats and pigs relaxin levels are elevated and keep rising until parturition, when levels fall down. ${ }^{2}$ In late pregnancy in mammals, in second half of gestation, relaxin is primarily secreted by placenta. ${ }^{4}$

Relaxin plays a role in uterine contractility and growth, being able to inhibit myometrial contractility in guinea pigs, pigs, mice, rats and hamsters. This hormone inhibits contractility in the first half of rat pregnancy while in the second half of pregnancy there is no inhibition..$^{2,4}$ In humans, this effect is very little and is not observed ${ }_{2,4}$ perhaps by the action of progesterone, which is involved in the inhibition of myometrial contractility and compete with relaxin in relation with this role. Relaxin participates in cervix growth in humans, which is mediated by an increase in prostromelysin, (proMMP-1, proMMP-2) and in procollagenase. ${ }^{4}$

Another remarkable function of relaxin in reproductive tissues is the angiogenesis process, which is mediated by the vascular endothelial growth factor (VEGF). In humans, relaxin induces the secretion of VEGF by human endometrial cells and in endometrial stromal and glandular epithelium cells collected from de secretory phase of the menstrual cycle. Goldsmith et al., ${ }^{5}$ provided human relaxin-2 (and also exogenous progesterone and estradiol to stimulate menstrual cycle) to ovariectomized rhesus monkeys and they showed an increase in the number of arterioles as seen in morphological 
studies (the hematoxylin/eosin-stained sections); a prominent increase in uterine weight, which was determined by the weight of the entire uterus after monkeys sacrification; and an increase in the tissue inhibitor of metalloproteinase 1 levels, determined by Western Blot assays. Relaxin's role in angiogenesis remains unclear due to the fact that Marshall et al., ${ }^{6}$ studies with relaxin deficient mouse (Rln -) showed a significant increase in the vascular endothelial growth factor A genes ( $\operatorname{Veg} f A$ ) but this fact did not show a significant increase in blood vessels proliferation. These studies also showed that in $R l n$ - mice, a significant decrease in endometrial angiogenesis were not seen. ${ }^{6}$ In conclusion, endogenous relaxin is not inducing angiogenesis in mouse endometrial tissue. ${ }^{6}$ In the cardiovascular system, relaxin carries out typical pregnancy modifications that include a decrease in blood pressure and an increment in the heart rate, plasma volume and cardiac output. $^{2}$

Although relaxin is important in softening pubic ligament, it is suggested that is not indispensable for this role because hyporelaxinemic women (without corpus luteum) are able to give birth without serious problems. Nevertheless, a relaxin knockout mouse model experience deficient development of mammary gland, the pubic symphysis and nipples, and has an atrophic vaginal and cervical luminal epithelium during pregnancy. ${ }^{4}$ The role of relaxin expression in the placental tissues in humans is not clear, but in other mammals, the relaxin family peptide known as INSL3 (insulin-like peptide 3 ) is expressed in the chorion villi that anchor the endometrium and participates in the nutrient exchange, and is also expressed in the maternal blood vessels. ${ }^{2}$

\section{Relaxin and reproduction in males}

Northern blotting, RT-PCR (reverse-transcription/polymerase chain reaction), immunohistochemical and immunoreactive studies have recognized relaxin mRNA in the glandular epithelium of the prostate, the glandular epithelium of the seminal vesicles, the ampulla of the vas deferens and also in the seminal plasma., ${ }^{2,7-9}$

The relaxin/insulin-like family peptide receptor 1 (RXFP1) is expressed on ejaculated human spermatozoa, where it can mediate the acrosome reaction, decrease apoptosis, increase mitochondrial activity, hyperactivation, calcium and cAMP (cyclic adenosine monophosphate) levels and mediate penetration into oocytes. ${ }^{2,10}$ RXFP1 is also expressed in spermatocytes and spermatids and Leydig cells, and is implicated in sperm motility and fertilizing ability in human spermatozoa. ${ }^{11}$ Relaxin is able to promote Sertoli cells proliferation through the activation of the PI3K/AKT (phosphatidylinositol-3kinase/protein kinase B) and MEK/ERK1/2 (Mitogen-activated protein kinase kinase/ extracellular signal-regulated kinases 1/2) pathways. ${ }^{11}$ Related to the prostate, relaxin-2 is capable to regulate the expression of prostate-specific antigen by the activation of the PI3K/ $\mathrm{Akt} / \mathrm{NF \kappa B}$ (nuclear factor kappa-light-chain-enhancer of activated $\mathrm{B}$ cells) and components of $\beta$-catenin/Wnt signaling. ${ }^{10,12}$ Moreover, in neuroendocrine differentiation of prostate cells, there is a raise in relaxin-2 expression. ${ }^{10,13}$

Deficiency of relaxin in males remains controversial: Samuel et al., ${ }^{14}$ studied $R l n^{-/}$male mice and wild-type $R l n^{+/+}$and found a decrease in prostate, testis epididymis and seminal vesicles weight in $R l n^{-/}$mice. They also showed a decreased epithelial proliferation in the prostate and decreased sperm maturation. ${ }^{14}$ These studies suggested that relaxin lack could produce local fibrosis in the prostate gland and in testes, but the following study by Ganesan et al. [15] do not observe the same results. Ganesan et al., ${ }^{15}$ treated $R l{ }^{-1}$ male mice with relaxin and they did not observe changes in prostate morphology. Finally, it is not demonstrated that the relaxin plays a fundamental role in male reproductive organ development.

\section{Conclusion}

Relaxin plays a potential role in reproduction in both females and males, especially in parturition and in spermatogenesis, respectively. Although relaxin reproductive function is the most well-known, there are aspects that remain unclear and there is the need to continue the study of the mechanisms and metabolic pathways that relaxin promote in order to understand better its potential role in reproduction. For example, there is little knowledge about the physiological role of relaxin in male reproduction and there is not much information about the pathways in which relaxin participates in female parturition.

\section{Acknowledgements}

None.

\section{Conflicts of interest}

The author declares there is no conflict of interest.

\section{References}

1. Bartsch O, Bartlick B, Ivell R. Relaxin signalling links tyrosine phosphorylation to phosphodiesterase and adenylyl cyclase activity. Mol Hum Reprod. 2001;7(9):799-809.

2. Bathgate RA, Halls ML, van der Westhuizen ET, et al. Relaxin family peptides and their receptors. Physiol Rev. 2013;93(1):405-480.

3. Vannuccini S, Bocchi C, Severi FM, et al. Endocrinology of human parturition. Ann Endocrinol (Paris). 2016;77(2):105-113.

4. Klein C. The role of relaxin in mare reproductive physiology: A comparative review with other species. Theriogenology. 2016;86(1):451-456.

5. Goldsmith LT, Weiss G, Palejwala S, et al. Relaxin regulation of endometrial structure and function in the rhesus monkey. Proc Natl Acad Sci US A. 2004;101(13):4685-4689.

6. Marshall SA, Ng L, Unemori EN, et al. Relaxin deficiency results in increased expression of angiogenesis- and remodelling-related genes in the uterus of early pregnant mice but does not affect endometrial angiogenesis prior to implantation. Reproductive, Biology and Endocrinology. 2006;14:1-11.

7. Loumaye E, De Cooman S, Thomas K. Immunoreactive relaxinlike substance in human seminal plasma. J Clin Endocrinol Metab. 1980;50(6):1142-1143.

8. Sokol RZ, Wang XS, Lechago J, et al. Immunohistochemical localization of relaxin in human prostate. Journal of Histochemistry \& Cytochemistry. 1989;37(8):1253-1255.

9. Gunnersen JM, Fu P, Roche PJ, Tregear GW (1996) Expression of human relaxin genes: characterization of a novel alternatively-spliced human relaxin mRNA species. Mol Cell Endocrinol 118(1-2): 85-94.

10. Ivell R, Agoulnik AI, Anand-Ivell R. Relaxin-like peptides in male reproduction-a human perspective. Br J Pharmacol. 2006;174(10):9901001.

11. Pimenta MT, Francisco RAR, Silva RP, et al. Relaxin affects cell organization and early and late stages of spermatogenesis in a coculture of rat testicular cells. Andrology. 2015;3(4):772-786.

12. Liu S, Vinall RL, Tepper C, et al. Inappropriate activation of androgen receptor by relaxin via beta-catenin pathway. Oncogene. 2008;27(4):499-505. 
13. Figueiredo KA, Palmer JB, Mui AL, et al. Demonstration of upregulated $\mathrm{H} 2$ relaxin mRNA expression during neuroendocrine differentiation of LNCaP prostate cancer cells and production of biologically active mammalian recombinant 6 histidine-tagged $\mathrm{H} 2$ relaxin. Ann N Y Acad Sci. 2005;1041:320-327.

14. Samuel CS, Tian H, Zhao L, et al. Relaxin is a key mediator of prostate growth and male reproductive tract development. Laboratory Investigaction. 2003;83(7):1055-1067.
15. Ganesan A, Klonisch T, McGuane JT, et al. Normal prostate morphology in relaxin-mutant mice. Reprod Fertil Dev. 2009;21(3):440-450. 